\title{
Amplitude based feedback control for NTM stabilisation at ASDEX Upgrade
}

\author{
Christopher Rapson ${ }^{\mathrm{a}}$, Louis Giannone ${ }^{\mathrm{a}}$, Marc Maraschek ${ }^{\mathrm{a}}$, Matthias Reich ${ }^{\mathrm{a}}$, Joerg Stober ${ }^{\mathrm{a}}$, Wolfgang Treutterer ${ }^{\mathrm{a}}$, the \\ ASDEX Upgrade Team ${ }^{\mathrm{a}}$
}

${ }^{a}$ Max Planck Institute for Plasma Physics, EURATOM Association, Boltzmannstrasse 2, 85748 Garching, Germany

\begin{abstract}
Neoclassical Tearing Modes (NTMs) degrade the confinement in tokamak plasmas at high beta, placing a major limitation on the projected fusion performance. Furthermore, NTMs can lead to disruptions with even more severe consequences. Therefore methods to stabilise NTMs are being developed with high priority at several research institutes worldwide. The favoured method is to deposit Electron Cyclotron Current Drive (ECCD) precisely at the mode location by controlling a movable mirror in the ECCD launcher. This method requires both the mode location and the deposition location to be known with high accuracy in real time. The required accuracy is given by half of the marginal island width, or approximately $1 \mathrm{~cm}$ for a $\mathrm{m} / \mathrm{n}=3 / 2 \mathrm{NTM}$ at ASDEX Upgrade. Despite considerable development on a range of diagnostics, it remains challenging to provide the necessary accuracy reliably and in real time.

To relax the accuracy requirements and add robustness, the feedback controller can additionally consider the effect of ECCD on the NTM amplitude directly. Then the optimal deposition location is simply where the NTM amplitude is minimised. The simplest implementation sweeps the ECCD beam across the expected NTM location. After the sweep, the beam can be returned to the optimal location and held there to stabilise the NTM. Unfortunately, waiting for a full sweep takes too long. Therefore a second method assesses the NTM growth every $100 \mathrm{~ms}$ and hones in on the NTM in a series of steps. The two algorithms employ similar principles to the "Target Lock" and "Search \& Suppress" algorithms implemented at DIII-D [1]. This paper presents the development of both algorithms using a simulation of the full feedback loop including NTM dynamics modelled by a Modified Rutherford Equation, and a demonstration of their effectiveness during ASDEX Upgrade experiments.
\end{abstract}

Keywords: NTM, neoclassical tearing mode, feedback control, ASDEX Upgrade, Modified Rutherford Equation

\section{Introduction}

Neoclassical Tearing Modes (NTM) are detrimental to confinement in tokamaks [2] and can even cause disruptions [3]. Therefore several groups worldwide are working on ways to stabilise these modes. The method of choice is localised Electron Cyclotron Current Drive (ECCD). One of the main challenges is the high accuracy required. To have a stabilising effect, ECCD must be deposited within the NTM island. The boundary case is a marginal width, below which the island is self-stabilising. On ASDEX Upgrade an NTM with poloidal/toroidal mode number $\mathrm{m} / \mathrm{n}=3 / 2$ has a marginal width of 2-2.5 cm [4] so the required accuracy is $\pm 1 \mathrm{~cm}$ in the radial direction.

There are several diagnostics available to detect and localise NTMs, but despite considerable recent devel-

Email address: chris.rapson@ipp.mpg.de (Christopher Rapson)

Preprint submitted to Fusion Engineering and Design opments, this kind of resolution remains challenging. Possibly the biggest challenge is to map measurements of the NTM at the midplane to the ECCD deposition location at the top of the flux surface. This is done using the real-time magnetic equilibrium reconstructions. Experience at ASDEX Upgrade has shown that without information on the internal current profile, it is not possible to fulfil these high demands [5]. The internal current profile can be obtained from Motional Stark Effect diagnostics, but this depends on the neutral beam configuration and a complex calibration procedure [e.g. 6]. Even if the equilibrium was known precisely, the NTM can be asymmetric [7], meaning that it does not lie on the equilibrium flux surface.

Model based profile estimation [8] or inline ECE [9] may give other options to improve the diagnostic situation in the near future. Alternatively, the controller can consider the effect of ECCD on the NTM amplitude directly. Then the optimal deposition location is simply

January 23, 2014 


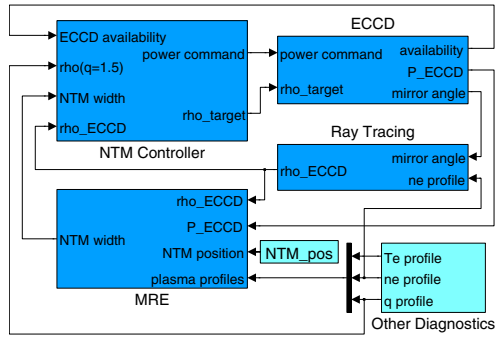

Figure 1: The model used to simulate the loop for amplitude based feedback control.

that which minimises the amplitude. DIII-D has had success with this technique in the past [1] and now two algorithms have been developed at ASDEX Upgrade to work along similar lines. This paper presents the two algorithms, their development using a simulation and successful implementation in experiments. Similarities and differences to the DIII-D algorithms will be noted.

NTM amplitude is usually defined in terms of the island width. In the experiment, magnetic fluctuations measured by Mirnov coils are taken as a proxy. Spatial filtering is applied to distinguish toroidal mode numbers and temporal filtering improves the signal to noise ratio.

\section{Simulation}

The simulation used to develop the algorithm is a combination of two existing simulations. One models the ECCD feedback control system [10] and another the NTM using a Modified Rutherford Equation (MRE) [4]. The combined model is shown in Fig. 1. Clockwise from the top, ECCD is launched with power $P_{E C C D}$ at a certain mirror angle. The deposition location $\rho_{E C C D}$ is calculated via ray-tracing. (In this paper, $\rho$ refers to rho poloidal, the normalised radial magnetic flux coordinate.) The MRE compares $\rho_{E C C D}$ to the island position, and calculates the evolution of the NTM amplitude in terms of island width, taking various plasma parameters into account. The cyan boxes represent data which are not generated within the loop, and may be synthesised or read in from measurement archives. Finally the NTM Controller uses the NTM amplitude to generate commands for the ECCD.

\section{Sweep \& Suppress}

The simpler of the two algorithms has been dubbed "Sweep \& Suppress". As for "Target Lock" at DIII$\mathrm{D}$ [1], the ECCD is swept across a region where the NTM is expected, and after the sweep it is targetted at the location which gave the lowest amplitude.
To start, the mirror is positioned such that $\rho_{E C C D}$ lies between the $\mathrm{q}=1.5$ and $\mathrm{q}=2.0$ surfaces, where the $3 / 2$ and 2/1 NTMs occur, respectively. When an NTM is detected, all available gyrotrons are swept across the relevant surface. The sweep takes one second, and extends 0.1 in $\rho$ on either side of the estimated flux surface. 0.1 in $\rho$ corresponds to $4 \mathrm{~cm}$ in the radial direction at the outboard midplane for this scenario. During the sweep, if the amplitude is the lowest so far, then the offset from the flux surface will be recorded. The offset is set to $\rho_{E C C D}-\rho_{q=1.5}$ from $200 \mathrm{~ms}$ before the minimum amplitude. $200 \mathrm{~ms}$ allows for the dynamics of the NTM ( $\tau \sim 100 \mathrm{~ms})$ and a low pass filter $(\tau=100 \mathrm{~ms})$.

As an additional condition, the amplitude of the NTM should be less than at the previous time step. Before the first comparison, the amplitude is initialised to $-\infty$. The condition will always be satisfied for any true minimum, and is used to guard against an NTM which is still growing at the beginning of the sweep. In this case the very first sample might have the lowest amplitude but will probably not be the optimal deposition location. If any gyrotrons trip during the sweep, then the sweep must be restarted with the remaining gyrotrons. If no gyrotrons are available, the algorithm will wait.

It is also possible to use the largest negative growth rate as an indication of optimal alignment, but simulations showed that taking the time derivative of the amplitude amplified the noise and the estimate of optimal alignment became less reliable.

Fig. 2 shows time traces from an experiment where this algorithm was used. The amplitude of a 3/2 NTM crosses the threshold at $2.3 \mathrm{~s}$ in frame a). When mirror control and gyrotron power are switched on at $3.0 \mathrm{~s}$ in frame d), the sweep starts in frame b). The optimal offset, which is continually updated in frame c), is found to be 0.03 inside the $\mathrm{q}=1.5$ surface. When ECCD is returned to this point, the amplitude is driven back down to the minimum, before recovering with $\beta$. The algorithm does not live up to the second part of its name here, but with only $660 \mathrm{~kW}$ ECCD in this scenario, that is to be expected.

"Target Lock" has additional features in that it will repeat the sweep if the amplitude does not drop below a threshold. This would be useful in case the first estimate is slightly off, or if the island moves after the sweep. In addition it builds a model of ECCD effectiveness based on the NTM growth rate at all positions. These features were not implemented for simplicity, and because the algorithm is intrinsically slow. During the sweep, stored energy will be lost from the plasma or the plasma could disrupt. This method is useful to demonstrate amplitude based feedback, but will not be developed further. 

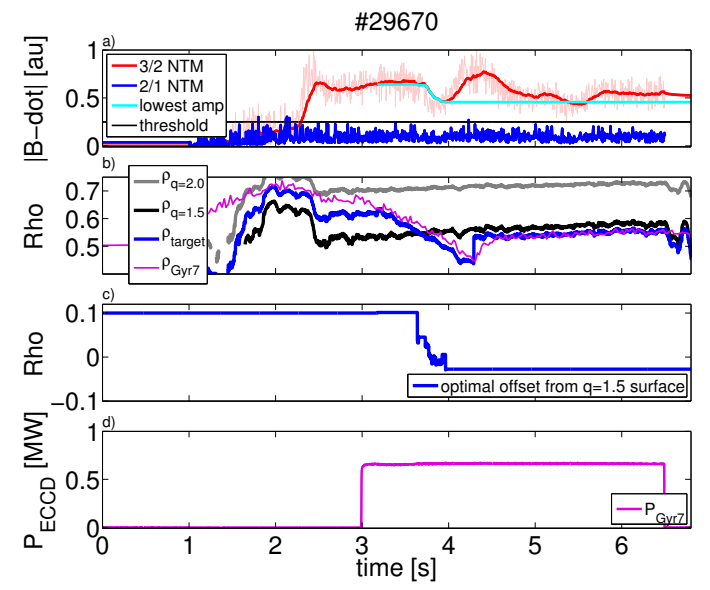

Figure 2: Time traces of real-time data recorded during shot \#29670. a) amplitudes of magnetic fluctuations from Mirnov coils, for even (pink=raw, red=filtered) and odd (blue=raw) toroidal mode numbers, with a threshold (black) and a record of the lowest amplitude during the sweep (cyan). b) Positions of rational flux surfaces, ECCD target and ECCD deposition from ray tracing. c) The controller's estimate of the optimal offset. d) Gyrotron power.

\section{Incremental Search}

The second algorithm was developed to locate the NTM without performing a full sweep. As for "Search \& Suppress" at DIII-D [1], ECCD is moved in a series of steps with a dwell time between each step. After the dwell time, the NTM amplitude and growth rate is evaluated to choose where the next step should be.

After detecting the presence and mode number of an NTM, this algorithm sets $\rho_{\text {target }}$ for all available gyrotrons to the relevant flux surface and then successively increments the offset. If the amplitude does not change significantly, the step will continue in the same direction as before. This is in order to keep scanning when the ECCD is far away and does not affect the NTM. If the amplitude decreases, the ECCD is close. If the growth rate is levelling off, going further might be a mistake so the controller holds the same offset. If the growth rate is becoming more negative, another step is taken.

If the amplitude increases and the growth rate is increasing, then ECCD is going in the wrong direction and will be turned around. If the amplitude increases but the growth rate is levelling off, changing direction might be a mistake. In this case it seems best to maintain the current offset and wait for more information in the next cycle.

Before taking any decision based on the amplitude, the algorithm checks that the mirror actuators are not at their limits and that the offset is $<0.1$ in $\rho$. In either of these cases the priority is to move away from the limit.

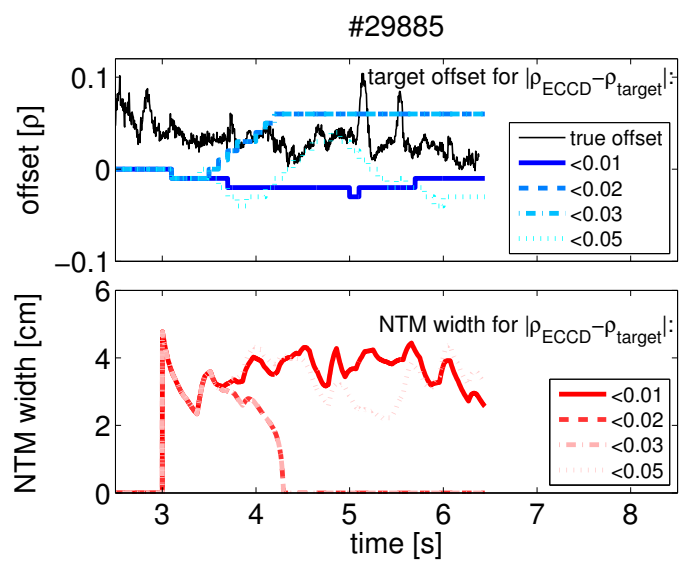

Figure 3: Simulation result using parameters from \#29885 showing the offset requested by the NTM Controller and the resulting NTM width for a series of allowed differences $\left|\rho_{E C C D}-\rho_{\text {target }}\right|$.

The simulation was used to test the stepping logic explained above, and to optimise several settings. For instance, it was found that shorter dwell times lead to more incorrect stepping decisions and finally take longer to stabilise the NTM. Longer dwell times delay the search, so a moderate value of $100 \mathrm{~ms}$ was selected. Also, due to slow mirror dynamics, changes to plasma parameters or signal noise, it is not necessarily true that $\rho_{E C C D}=\rho_{\text {target }}$. In order to base decisions on $\rho_{\text {target }}$, which is common to all gyrotrons, a maximum allowed difference $\left|\rho_{E C C D}-\rho_{\text {target }}\right|$ is set. If the limit is exceeded, the offset will not change. Results from simulations for a series of limits is shown in Fig. 3. If the limit is too small, $\rho_{E C C D}$ is often 'off-target' due to noise, and the target is changed very infrequently. If the limit is too large, several wrong decisions are made. In both cases it takes longer to stabilise the NTM. Both 0.02 and 0.03 gave good results (identical in the example shown) so the chosen setting was $\left|\rho_{E C C D}-\rho_{\text {target }}\right|<0.025$.

"Incremental Search" has performed well in experiments, as shown by two examples in fig. 4. Shot \#30057 demonstrates how the algorithm copes with unexpected events, such as gyrotron trips and a 2/1 NTM. Gyrotron 6 trips immediately in frame d), but the algorithm continues the search as long as one gyrotron is operational. Twice, the amplitude in frame a) is reduced when the algorithm finds the optimal position, but with $750 \mathrm{~kW}$ cannot shrink it below the marginal width. The controller then overshoots, the amplitude increases, and the steps change direction. At $3.3 \mathrm{~s}$ an example of a 'double direction change' can be seen, where the initial amplitude increase causes the first direction change at $3.2 \mathrm{~s}$. However, $100 \mathrm{~ms}$ later the NTM is still growing 

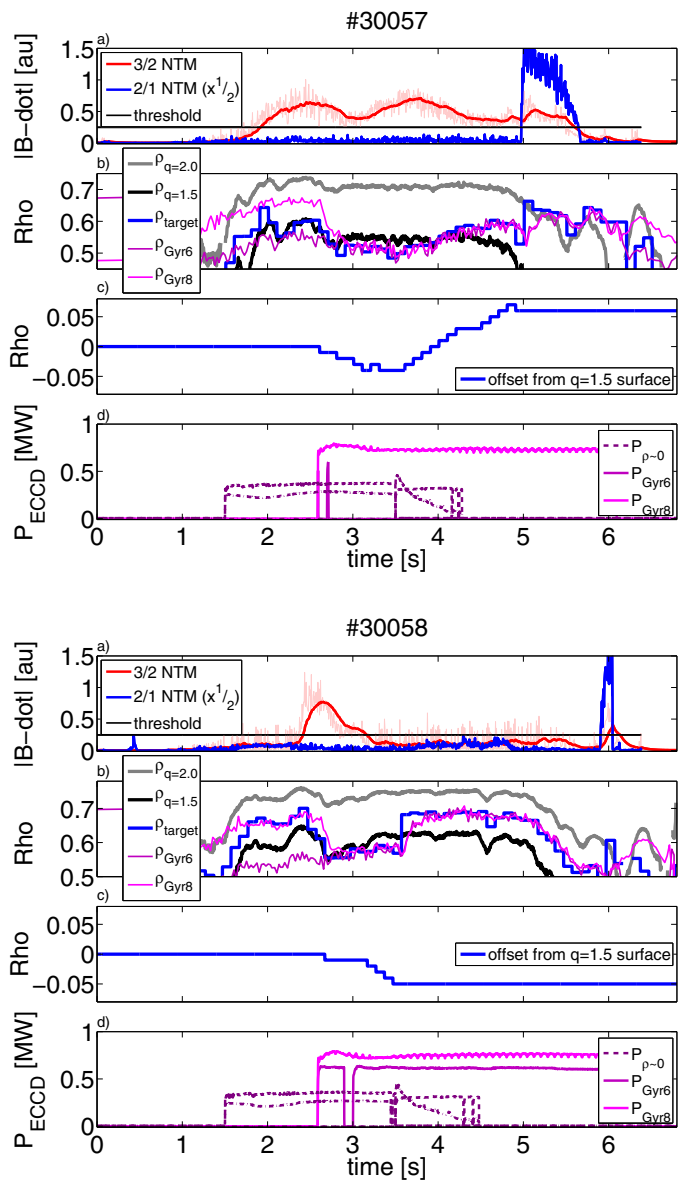

Figure 4: Experimental results using Incremental Search. Axes are as for Fig. 2.

and so the controller makes another direction change. This is the wrong decision in this case and increases the time required to return to the optimal position. Later in the shot, two more gyrotrons trip and the scenario changes. A 2/1 NTM is detected at $5.0 \mathrm{~s}$, and the controller switches to the new target. The offset in frame c) is now irrelevant. Along with radiation and $\beta$ losses caused by the NTM itself, ECCD contributes to stabilise the NTM before the heating rampdown at $6.0 \mathrm{~s}$

In shot \#30058 the location of the $q=1.5$ surface predicted by the equilibrium reconstruction accurately matches the NTM location, and the amplitude immediately decreases when the controller is switched on at $2.5 \mathrm{~s}$. The strongest decline is at the start, and after one step the controller maintains this offset until the rate of decline increases again. Once the NTM is stabilised, the controller moves ECCD back to its starting position between $\mathrm{q}=1.5$ and $\mathrm{q}=2.0$. Note that during a gyrotron trip from 2.9 to $3.0 \mathrm{~s}$ the amplitude is constant, confirming that ECCD is responsible for the stabilisation.

The stepping logic here is slightly different to "Search \& Suppress", in an effort to use fewer thresholds and thereby to make the algorithm more general. Similarly, using rational flux surfaces from the equilibrium as a starting point automatically adapts the algorithm to almost any plasma scenario. After stabilisation the controller saves the last offset, but does not try to perform preventive stabilisation, as the current strategy is to minimise reaction time for whichever NTM is triggered.

\section{Conclusions \& Future Work}

Two algorithms have been developed which use the NTM amplitude to find the optimal deposition location for ECCD, and thus reduce the accuracy demands on other diagnostics. The principle of amplitude based feedback was successfully demonstrated with "Sweep \& Suppress", and "Incremental Search" showed that it can stabilise an NTM on short time scales. Further optimisations will attempt to reduce overshoot, avoid 'double direction changes', and to integrate amplitude based feedback into the standard NTM Controller.

\section{Acknowledgements}

The authors would like to thank E. Kolemen for fruitful discussions regarding NTM stabilisation at DIII-D.

\section{References}

[1] D. A. Humphreys, et al., Active control for stabilization of neoclassical tearing modes, Phys. Plasmas 13 (2006).

[2] T. C. Hender, et al., Chapter 3: MHD stability, operational limits and disruptions, Nucl. Fusion 47 (2007) S128-S202.

[3] B. Esposito, et al., Disruption control on FTU and ASDEX upgrade with ECRH, Nucl. Fusion 49 (2009).

[4] L. Urso, Modelling and Experiments on NTM stabilisation at ASDEX Upgrade, Ph.D. thesis, Technische Universitaet Muenchen, 2009

[5] L. Giannone, et al., Real-time magnetic equilibria for preemptive NTM stabilization experiments on ASDEX Upgrade, Nucl. Fusion (2013). To be publishd.

[6] H. Yuh, The Motional Stark Effect Diagnostic on Alcator CMod, Ph.D. thesis, Massachusetts Institute of Technology, 2005.

[7] C. Ren, M. Chu, J. Callen, Magnetic island deformation due to sheared flow and viscosity, Phys. Plasmas 6 (1999) 1203-1207.

[8] F. Felici, et al., Real-time physics-model-based simulation of the current density profile in tokamak plasmas, Nucl. Fusion 51 (2011).

[9] B. A. Hennen, et al., Real-time control of tearing modes using a line-of-sight electron cyclotron emission diagnostic, Plasma Phys. Contr. Fusion 52 (2010).

[10] C. J. Rapson, et al., Simulation of feedback control system for NTM stabilisation in ASDEX Upgrade, Fusion Eng. Des. (2013). 